\title{
POLYCYCLIC AROMATIC HYDROCARBON FAR-INFRARED SPECTROSCOPY
}

\author{
C. Boersma ${ }^{1}$, C. W. Bauschlicher, JR. ${ }^{2}$, A. Ricca ${ }^{2,3}$, A. L. Mattioda ${ }^{1}$, E. Peeters ${ }^{3,4}$, A. G. G. M. Tielens ${ }^{1,5}$, \\ AND L. J. Allamandola ${ }^{1}$ \\ ${ }^{1}$ NASA Ames Research Center, MS 245-6, Moffett Field, CA 94035, USA; christiaan.boersma@nasa.gov \\ 2 NASA Ames Research Center, MS 230-3, Moffett Field, CA 94035, USA \\ 3 SETI Institute, 189 Bernardo Avenue, Suite 100, Mountain View, CA 94043, USA \\ ${ }^{4}$ Department of Physics and Astronomy, The University of Western Ontario, London, ON N6A 3K7, Canada \\ 5 Leiden Observatory, Leiden University, P.O. Box 9513, NL-2300 RA, The Netherlands \\ Received 2010 October 13; accepted 2011 January 5; published 2011 February 9
}

\begin{abstract}
The far-IR characteristics of astrophysically relevant polycyclic aromatic hydrocarbons (PAHs) averaging in size around 100 carbon atoms have been studied using the theoretical spectra in the NASA Ames PAH IR Spectroscopic Database. These spectra were calculated using density functional theory. Selections of PAH species are made, grouped together by common characteristics or trends, such as size, shape, charge, and composition, and their far-IR spectra compared. The out-of-plane modes involving the entire molecule are explored in detail, astronomical relevance is assessed, and an observing strategy is discussed. It is shown that PAHs produce richer far-IR spectra with increasing size. PAHs also produce richer far-IR spectra with increasing number of irregularities. However, series of irregular-shaped PAHs with the same compact core have common "Jumping-Jack" modes that "pile up" at specific frequencies in their average spectrum. For the PAHs studied here, around 100 carbon atoms in size, this band falls near $50 \mu \mathrm{m}$. PAH charge and nitrogen inclusion affect band intensities but have little effect on far-IR band positions. Detailed analysis of the two-dimensional, out-of-plane bending "drumhead" modes in the coronene and pyrene "families" and the one-dimensional, out-of-plane bending "bar" modes in the acene "family" show that these molecular vibrations can be treated as classical vibrating sheets and bars of graphene, respectively. The analysis also shows that the peak position of these modes is very sensitive to the area of the emitting PAH and does not depend on the particular geometry. Thus, these longest wavelength PAH bands could provide a unique handle on the size of the largest species in the interstellar PAH family. However, these bands are weak. Observing highly excited regions showing the mid-IR bands in which the emission from classical dust peaks at short wavelengths offers the best chance of detecting PAH emission in the far-IR. For these regions sensitivity is not an issue, spectral contrast is maximized and the PAH population is only comprised of highly stable, compact symmetric PAHs, such as the members of the pyrene and coronene "families" discussed in detail here.
\end{abstract}

Key words: astrochemistry - ISM: lines and bands - methods: laboratory - methods: numerical - molecular data - techniques: spectroscopic

Online-only material: color figures

\section{INTRODUCTION}

ESA's Herschel satellite and NASA's Stratospheric Observatory For Infrared Astronomy (SOFIA) are expanding our knowledge in the far-IR $(\lambda \gtrsim 20 \mu \mathrm{m})$. Laboratory experiments and density functional theory computations show that polycyclic aromatic hydrocarbon (PAH) molecules have bands that span the far-IR from 20 to $1000 \mu \mathrm{m}\left(500-1 \mathrm{~cm}^{-1} ; 15-0.03 \mathrm{THz}\right)$ and beyond (Moutou et al. 1996; Mulas et al. 2006a; Malloci et al. 2007; Mattioda et al. 2009; Ricca et al. 2010; Bauschlicher et al. 2010). Since the mid-IR spectrum of many astronomical objects is dominated by the well-known PAH bands, emission from these molecules should also contribute to their far-IR spectrum. Unlike the mid-IR, where fundamental vibrational frequencies are determined largely by vibrations involving the chemical subgroups and specific bonds which make up the molecule, these longer wavelength transitions originate in vibrations of the entire molecule. Thus, while the mid-IR spectra of PAHs resemble one another since all PAHs are part of a specific chemical class, beyond about the $15-20 \mu \mathrm{m}$ band, where the $\mathrm{C}-\mathrm{C}-\mathrm{C}$ bending modes start to populate the spectrum, positions depend on overall PAH shape and size.

Consequently, the far-IR spectra of astronomical objects showing the mid-IR PAH emission features will provide a very different perspective on molecular shape and size, which in turn, provide a new probe for local conditions, chemical history of carbonaceous material, etc. Furthermore, because the far-IR samples colder species than the mid-IR, these data will explore the large end of the astronomical PAH size distribution and expand the scope of astronomical and astrochemical regimes in which PAHs can serve as probes. Far-IR observations may even allow the identification of specific molecules in the interstellar PAH family (Mulas et al. 2006a; Tielens 2008).

Utilizing the theoretical spectra in the NASA Ames PAH IR Spectroscopic Database (Bauschlicher et al. 2010), an overview of the far-IR properties of several different PAH types is presented here. The fundamental modes governing the far-IR are assessed, with specific focus given to the modes involving the entire molecule. Because of their relevance in the astronomical context, emphasis is placed on PAHs of about 100 carbon atoms in size, as they are most likely to dominate far-IR astronomical spectra. The results are put in astronomical context and related to the earlier work and observing strategy proposed by Mulas et al. (2006a), based on the PAH database described in Malloci et al. (2007).

This work is presented as follows. In Section 2, the NASA Ames PAH IR Spectroscopic Database is briefly summarized, followed by a comparison of mid- and far-IR spectroscopic 


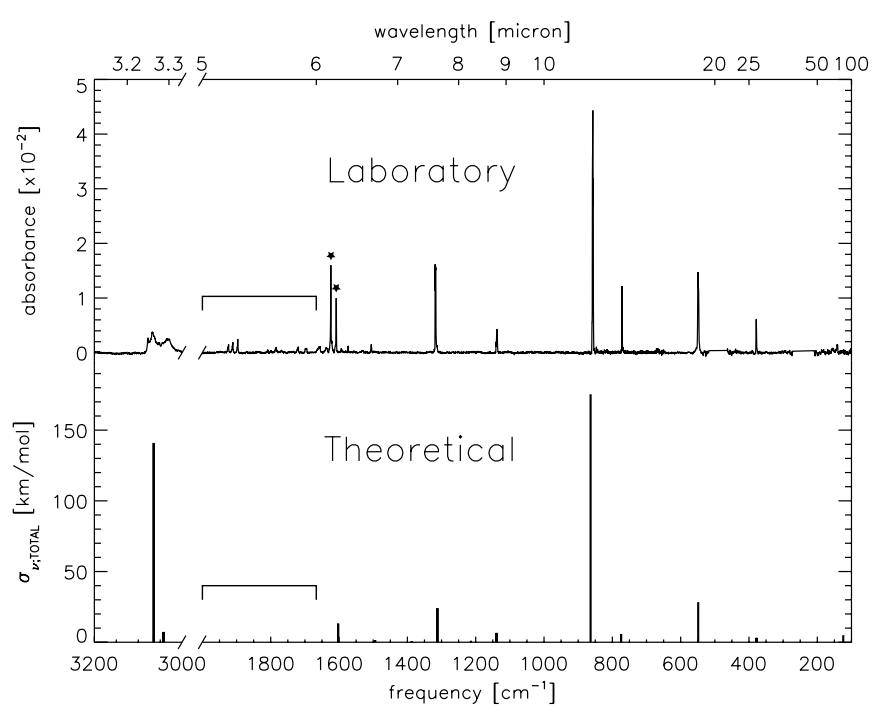

Figure 1. Full IR vibrational absorption spectrum of coronene computed using density functional theory (theoretical) compared to its experimentally measured absorption spectrum in an argon matrix (laboratory). The horizontal bracket indicates the 5-6 $\mu \mathrm{m}$ region. Bands here are due to overtone and combination modes, for which no computed data are available. Note: (1) details on the density functional theory calculations can be found in Bauschlicher et al. (2010); (2) the two stars in the laboratory spectrum indicate contributions from matrix isolated water impurities; (3) the experimental data below $650 \mathrm{~cm}^{-1}$ have been reproduced from Mattioda et al. (2009).

properties, the description of the typical molecular vibrations in the far-IR and the far-IR absorption spectra as a function of size, shape, charge, and nitrogen incorporation is presented. In Section 3, the out-of-plane bending "drumhead" modes in the coronene and pyrene "families" and the out-of-plane bending "bar" modes in the acene "family" are characterized using classical plate theory. This is followed in Section 4 by a discussion of the photophysics of the emission process, astronomical implications, and an observing strategy. The paper finishes in Section 5 with a summary and conclusions.

\section{FAR-IR PAH SPECTROSCOPY}

The NASA Ames PAH IR Spectroscopic Database consists of two parts: a collection of experimental spectra and a collection of theoretical spectra. Here, version 1.11 of the theoretical database is utilized, which contains 575 vibrational spectra spanning $2.7 \mu \mathrm{m}$ to $3.7 \mathrm{~mm}\left(3700-2.7 \mathrm{~cm}^{-1} ; 110-0.08 \mathrm{THz}\right)$, computed using density functional theory. These data, described in detail by Bauschlicher et al. (2010), are available at http://www.astrochem.org/pahdb.

Figure 1 compares the full IR vibrational spectrum of neutral coronene computed using density functional theory at the B3LYP level to its matrix isolated spectrum. The figure demonstrates the good agreement between the theoretically computed and experimentally determined band positions over the entire frequency range.

Figure 2 shows the mid- and far-IR absorption spectra of several PAH cations. Cations have been chosen because of the better spectral match with the astronomical 5-9 $\mu \mathrm{m}$ region. However, this does not exclude the presence of neutral PAHs in space. The figure demonstrates the striking qualitative difference between the mid- and far-IR spectra of PAHs. Unlike the midIR, where bands are produced by vibrations of the distinct chemical subgroups comprising the molecule, $\mathrm{PAH}$ vibrations that produce bands in the far-IR arise from motions that involve the molecule acting as a whole. Consequently, instead of bands falling in specific and distinct mid-IR wavelength regions corresponding to, say, $\mathrm{C}-\mathrm{C}$ or $\mathrm{C}-\mathrm{H}$ stretching and bending vibrations, other distinctions need to be made.

Mattioda et al. (2009) and Ricca et al. (2010) classified some of the typical PAH far-IR modes. Although there is an overlap of ranges in larger PAH molecules, for PAHs of about 100 carbon atoms in size these fall, more-or-less, in the following wavelength ranges:

“Jumping-Jack” modes. From about 20 to $130 \mu \mathrm{m}$ (15-2.2 THz; $500-75 \mathrm{~cm}^{-1}$ ) the bands originate from so-called "JumpingJack" modes, where the molecules deform in-plane around its central core and symmetrically around one axis.

"Butterfly" modes. From about 200 to $450 \mu \mathrm{m}$ (50-22 THz; $1.5-0.65 \mathrm{~cm}^{-1}$ ) the modes show similarities to a butterfly flapping its wings. The molecule makes a synchronized, outof-plane flapping motion around a central symmetry axis.

"Drumhead" modes. Beyond about $130 \mu \mathrm{m}\left(2.2 \mathrm{THz} ; 75 \mathrm{~cm}^{-1}\right)$ the lowest "drumhead" modes populate the spectrum. Similar to percussion instruments, the molecular PAH "sheet" makes an out-of-plane wave motion. This title is somewhat misleading however, since unlike drums, the edges of PAHs are not clamped but free.

\subsection{PAH Size}

Figure 3 compares the far-IR spectrum of the different charge states of naphthalene to that of increasingly larger acenes up to five ringed pentacene. The figure shows that the most prominent band in all neutral acenes studied to date falls between 21 and $22 \mu \mathrm{m}$ ( 14.4 and $13.8 \mathrm{THz} ; 480$ and $\left.460 \mathrm{~cm}^{-1}\right)$, shifting to slightly longer wavelength with increasing length. For all the cations the band shifts to about $22.5 \mu \mathrm{m}\left(13.6 \mathrm{THz} ; 445 \mathrm{~cm}^{-1}\right)$. For the two anions studied the longer wavelength bands increase in intensity.

Figure 4 shows the far-IR spectra of compact symmetric PAHs ranging in size from $\mathrm{C}_{24} \mathrm{H}_{12}$ to $\mathrm{C}_{130} \mathrm{H}_{28}$ in the anion, neutral, and cation forms. The mid-IR properties of $\mathrm{C}_{54} \mathrm{H}_{18}$ and larger PAHs are described in detail by Bauschlicher et al. (2008).

The figure demonstrates that the far-IR spectra of PAHs depend on shape. Although there are some exceptions, for the most part, going from the cation to neutral forms does not significantly alter the spectrum for a particular PAH. However for the PAH anions, only a few bands dominate the spectrum. The spectra tend to get richer in features and extend further into the far-IR with increasing molecular size for these compact molecules.

\subsection{Shape}

Figure 5 explores the influence of modifying the linear fused ring system of the acenes. The introduced structural changes can be viewed in two ways, either as the addition of an extra ring above and below the linear skeleton or as the substitution of the PAH pyrene in the linear fused ring system. Figure 5 compares the spectrum of pyrene with the spectra of several pyrenesubstituted acenes in the anion, cation, and neutral charge states. Comparing this figure to the spectra in Figure 3 shows that the prominent band between 21 and $23 \mu \mathrm{m}(14.3$ and $13 \mathrm{THz}$; 478 and $435 \mathrm{~cm}^{-1}$ ), which appears to be a characteristic of the linear acenes, is only important in the neutral pyrene-substituted species. The spectra of the anion and cation counterparts are significantly richer. Interestingly, while the intensity of the anion 


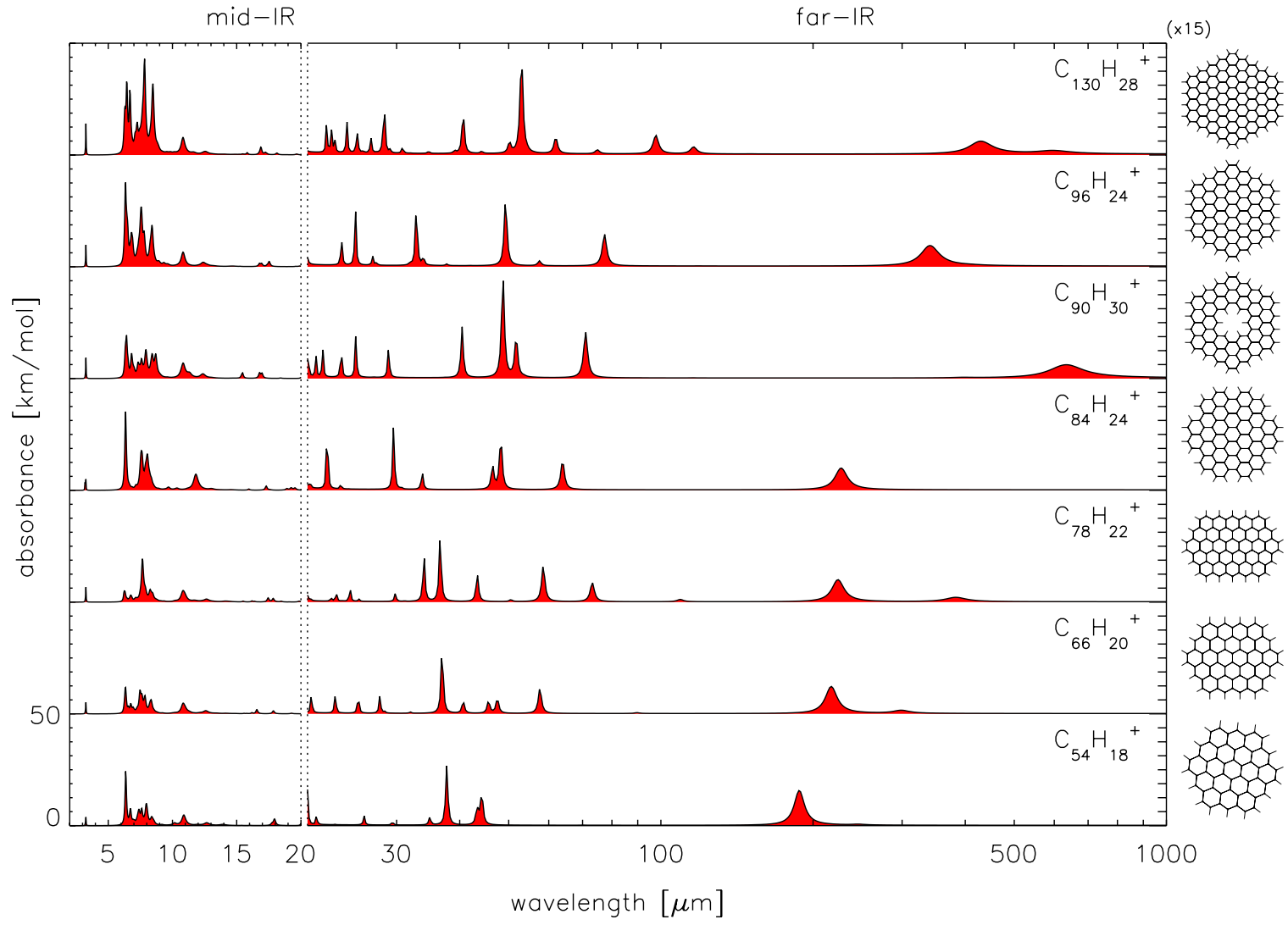

Figure 2. Computed mid- and far-IR $(2-1000 \mu \mathrm{m})$ absorption spectra of several large PAH cations. The far-IR has been presented on a logarithmic wavelength scale and the band intensities have been enhanced by a factor of 15 . Bands have been given Lorentzian profiles with an FWHM of 30, 6, and 3 $\mathrm{cm}^{-1}$ for the 2.5-15, 15-20, and $20-1000 \mu \mathrm{m}$ regions, respectively (see Section 4.1 for a discussion on the chosen band widths).

(A color version of this figure is available in the online journal.)
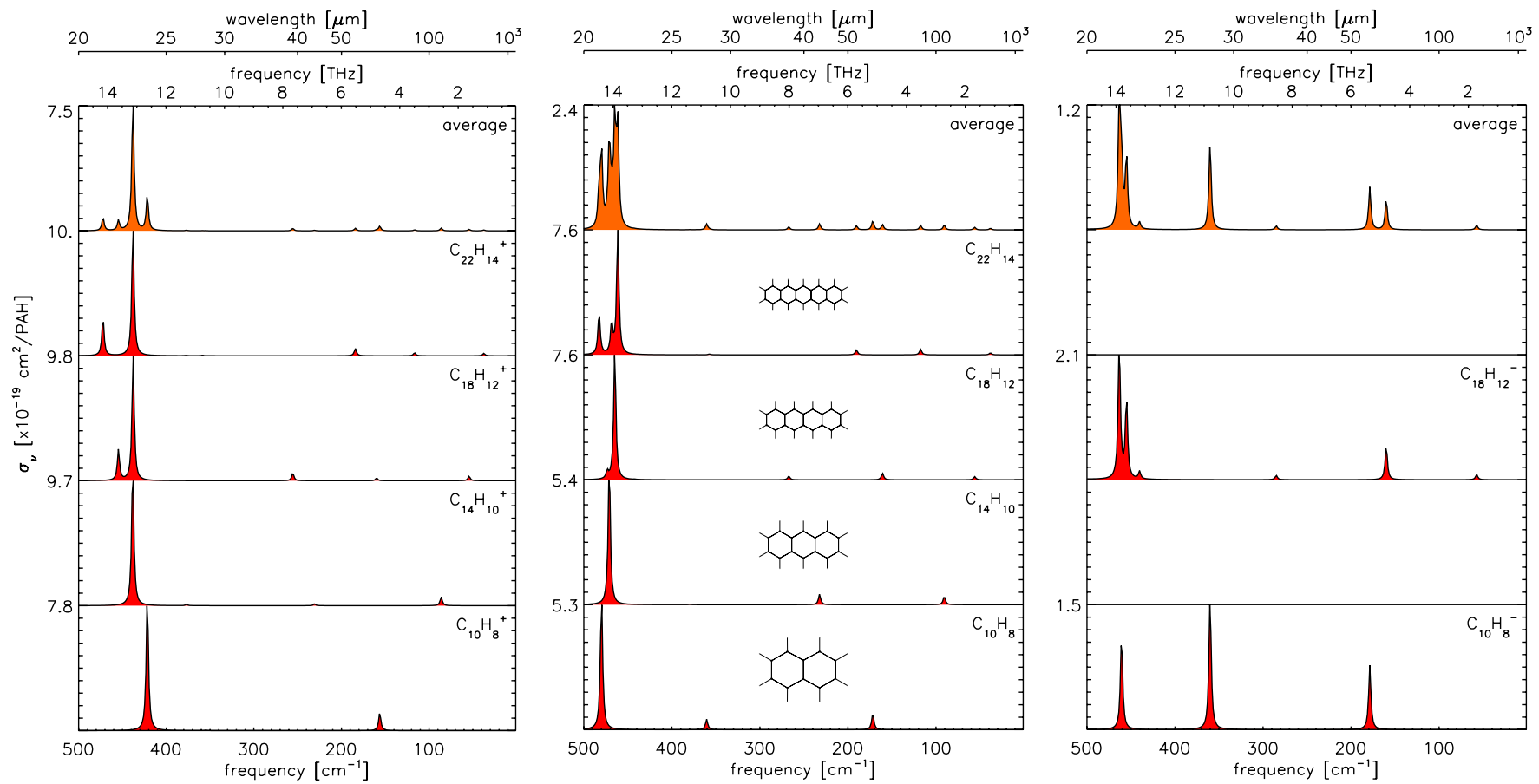

Figure 3. Computed far-IR $\left(500-1 \mathrm{~cm}^{-1} ; 15-0.03 \mathrm{THz} ; 20-10,000 \mu \mathrm{m}\right)$ absorption spectra of acenes up to five rings in their neutral and singly charged states and their equally weighted averages. Bands have been given Lorentzian profiles with an arbitrary FWHM of $3 \mathrm{~cm}^{-1}$. Note: there are no data on $\mathrm{C}_{14} \mathrm{H}_{10}^{-}$and $\mathrm{C}_{22} \mathrm{H}_{14}^{-}$in the database.

(A color version of this figure is available in the online journal.) 

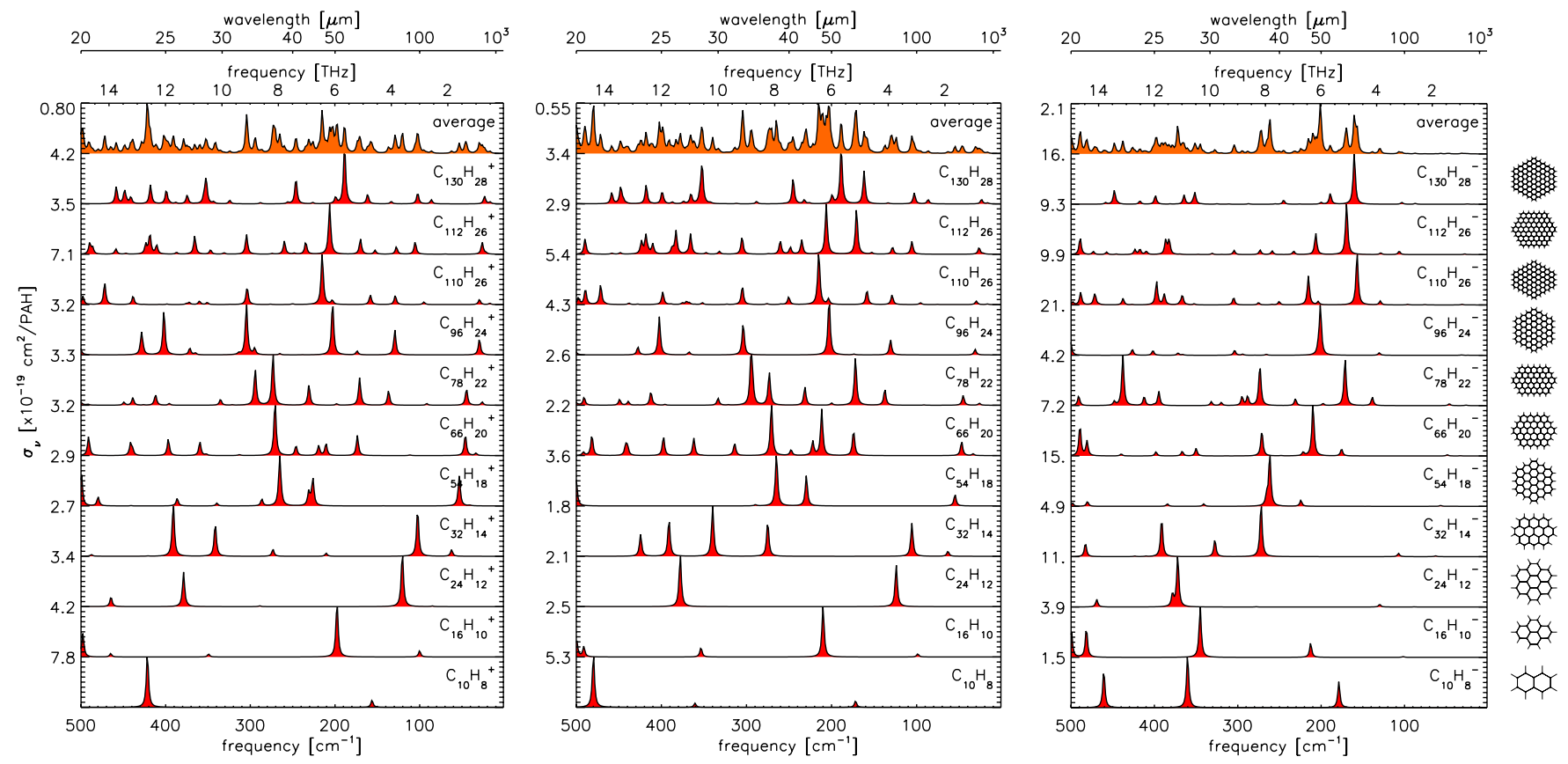

Figure 4. Computed far-IR $\left(500-1 \mathrm{~cm}^{-1} ; 15-0.03 \mathrm{THz} ; 20-10,000 \mu \mathrm{m}\right)$ absorption spectra for a series of compact PAHs in their neutral and singly charged states and their equally weighted averages. Bands have been given Lorentzian profiles with an arbitrary FWHM of $3 \mathrm{~cm}^{-1}$.

(A color version of this figure is available in the online journal.)
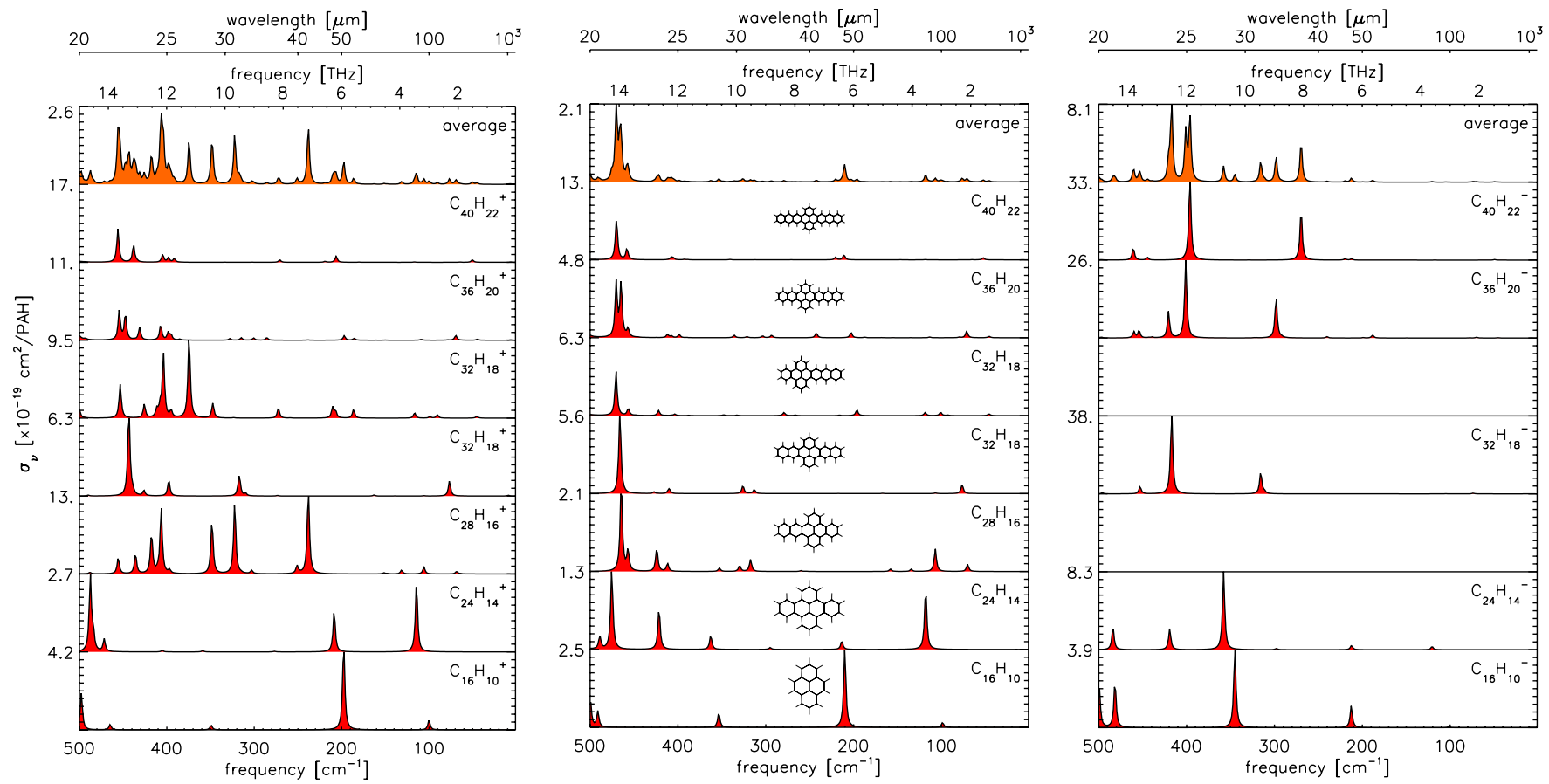

Figure 5. Computed far-IR $\left(500-1 \mathrm{~cm}^{-1} ; 15-0.03 \mathrm{THz} ; 20-10,000 \mu \mathrm{m}\right)$ absorption spectrum of pyrene compared to that of pyrene-substituted acenes in their neutral and singly charged states and together with their equally weighted averages. Bands have been given Lorentzian profiles with an arbitrary FWHM of $3 \mathrm{~cm}^{-1}$. Note: there are no data on the anion forms of $\mathrm{C}_{28} \mathrm{H}_{16}$ and $\mathrm{C}_{32} \mathrm{H}_{18}$ in the database.

(A color version of this figure is available in the online journal.)

forms in the acenes is slightly lower than the other forms, this is not the case for the substituted acenes, where the opposite holds.

Figure 6 illustrates the influence of PAH shape on the far-IR spectrum by investigating the effect of structural modifications on circumcircumcoronene $\left(\mathrm{C}_{96} \mathrm{H}_{24}\right)$. These include both the removal and addition of (multiple) rings. The mid-IR properties of these PAHs are described by Bauschlicher et al. (2009). The figure illustrates that with breaking molecular symmetry, PAH spectra get richer in features. The spectra have some bands in common, most noteworthy the feature around $50 \mu \mathrm{m}(6 \mathrm{THz}$; $200 \mathrm{~cm}^{-1}$ ). Analysis of the involved molecular vibrations shows that they are all due to the same "Jumping-Jack" mode and are doubly degenerate for the symmetric species. Interestingly 

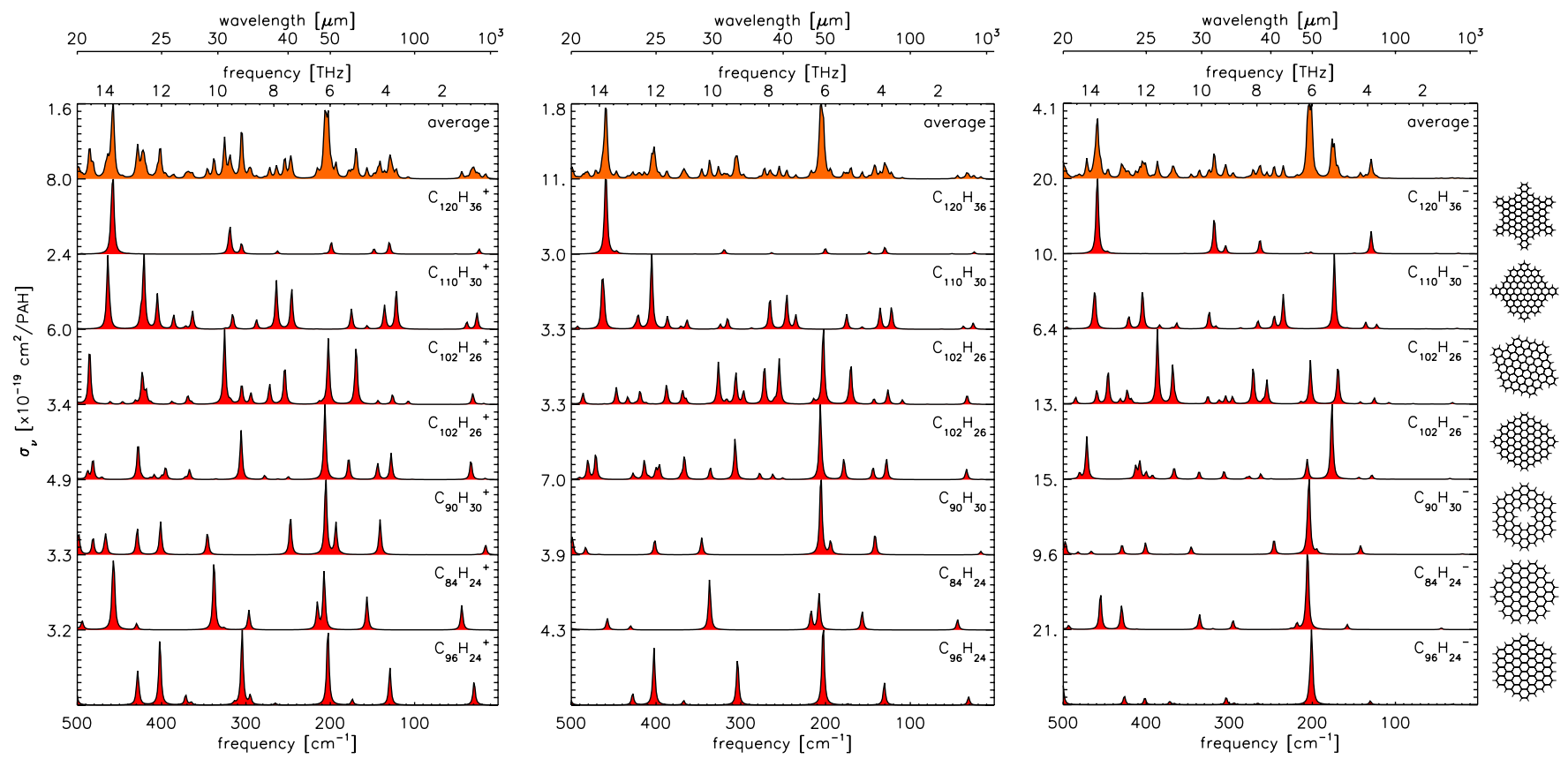

Figure 6. Computed far-IR absorption spectra $\left(500-1 \mathrm{~cm}^{-1} ; 15-0.03 \mathrm{THz} ; 20-10,000 \mu \mathrm{m}\right)$ for a series of irregular-shaped, large, PAHs in their neutral and singly charged states and their equally weighted averages. Bands have been given Lorentzian profiles with an arbitrary FWHM of $3 \mathrm{~cm}^{-1}$.

(A color version of this figure is available in the online journal.)
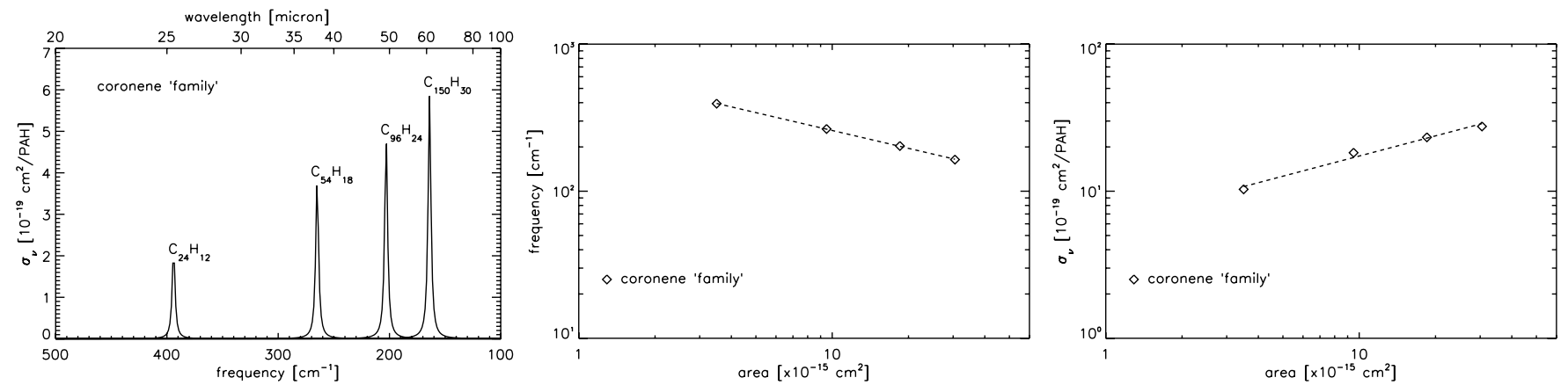

Figure 7. Left: the shift of the "Jumping-Jack" mode to lower frequencies as molecules in the coronene "family" (see Figure 10) increase in size. Bands have been given Lorentzian profiles with an arbitrary FWHM of $3 \mathrm{~cm}^{-1}$. Middle: the shift of the "Jumping-Jack" mode to lower frequencies as a function of area. The dashed line shows the least-squares fit represented by Equation (1). Right: the increase in intensity of the "Jumping-Jack" mode as a function of area. The dashed line shows the least-squares fit represented by Equation (2).

enough, addition of the two opposing pendent rings in $\mathrm{C}_{110} \mathrm{H}_{30}$ splits the degeneracy into two distinct bands. The pendent rings break the molecular symmetry and act as counterweights suppressing the molecular vibration. Furthermore, the two molecules with pendent rings produce a band between about 21 and $23 \mu \mathrm{m}$ (14.3 and $13 \mathrm{THz} ; 478$ and $\left.435 \mathrm{~cm}^{-1}\right)$, similar to the far simpler acenes in Figure 12. Analysis of the modes in the two irregular PAHs and the acenes shows that they are due to the same out-of-plane vibration across a single ring. Therefore, all species with single rings, such as pendent rings or the terminating rings of the acenes, should have this band.

Figure 7 shows how the "Jumping-Jack" feature in the coronene "family" (see Figure 10) shifts to longer wavelength and gets more intense with increasing PAH size. The figure also reveals that the frequencies scale with PAH area $(A)$ and are well fitted by the expression

$$
v=655\left(\frac{10^{-15}\left(\mathrm{~cm}^{2}\right)}{A}\right)^{2 / 5}\left(\mathrm{~cm}^{-1}\right)
$$

where $A$, the PAH surface area, is determined as $5 \times 10^{-16} \mathrm{~cm}^{2}$ per ring. The last panel of Figure 7 reveals that the integrated cross-section also scales with PAH area $(A)$ and are well fitted by the expression

$$
\sigma_{v, \text { int }}=6.1\left(\frac{A}{10^{-15}\left(\mathrm{~cm}^{2}\right)}\right)^{0.46}\left(\times 10^{-19} \mathrm{~cm}^{2}\right) .
$$

Considering Figure 4, besides members of the coronene "family," the spectra of some of the other compact symmetric PAHs also have the prominent band near $50 \mu \mathrm{m}\left(6 \mathrm{THz} ; 200 \mathrm{~cm}^{-1}\right)$.

Comparison of the anion forms shows that the absolute intensity of the modified $\mathrm{C}_{96} \mathrm{H}_{24}^{-}$molecules is only slightly weaker than that of $\mathrm{C}_{96} \mathrm{H}_{24}^{-}$itself. In combination with Figure 8, this suggests that the anion forms of the $\mathrm{C}_{96} \mathrm{H}_{24}$ class of molecules are particularly strong.

Ricca et al. (2010) studied the far-IR spectra of neutral PAHs ranging in size from 82 up to 130 carbon atoms and with compact shapes from round to oval and from rectangular to trapezoidal. Their work shows that the out-of-plane modes 

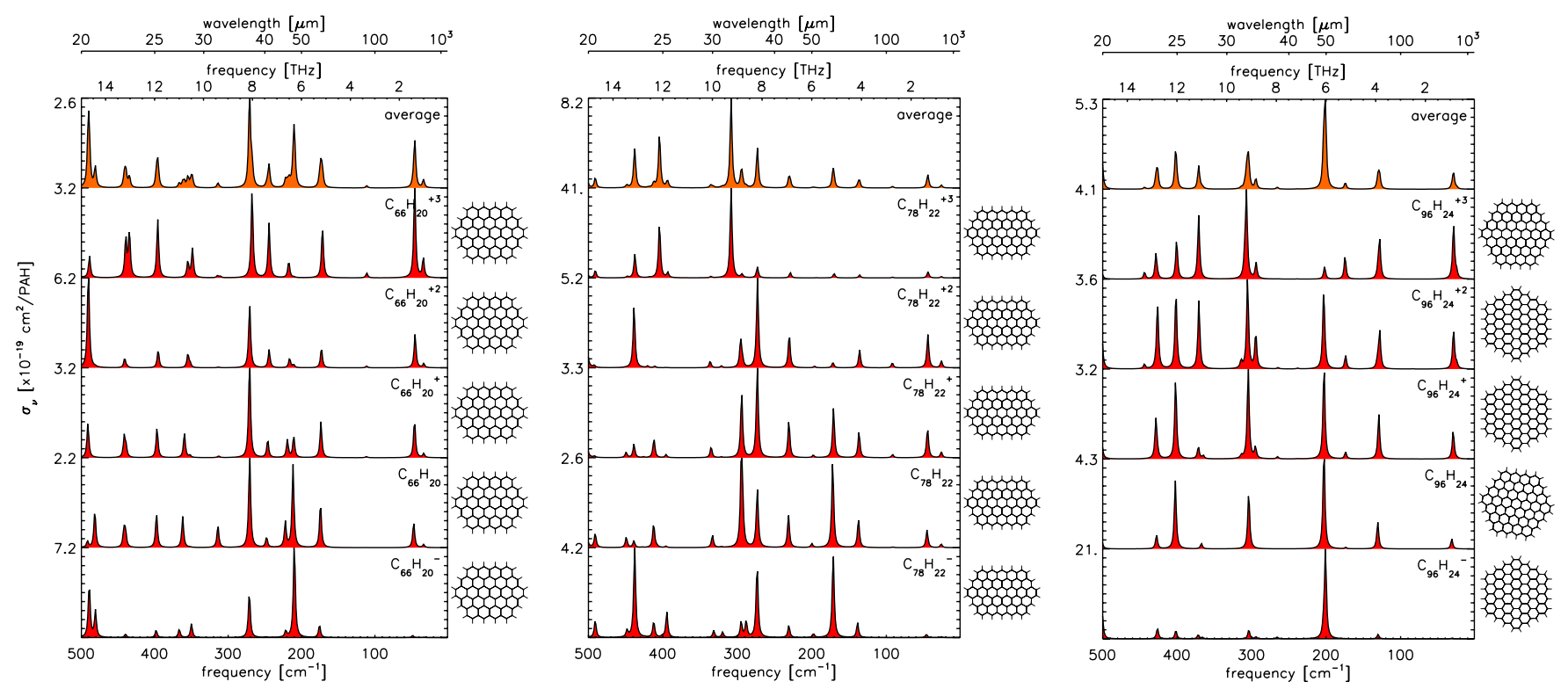

Figure 8. Computed far-IR $\left(500-1 \mathrm{~cm}^{-1} ; 15-0.03 \mathrm{THz} ; 20-10,000 \mu \mathrm{m}\right)$ absorption spectra of three large, compact PAHs $\left(\mathrm{C}_{66} \mathrm{H}_{20}, \mathrm{C}_{78} \mathrm{H}_{22}, \mathrm{C}_{96} \mathrm{H}_{24}\right)$ in their $-1,0$, $+1,+2$, and +3 charge states and their equally weighted averages. Bands have been given Lorentzian profiles with an arbitrary FWHM of $3 \mathrm{~cm}^{-1}$.

(A color version of this figure is available in the online journal.)

beyond $\sim 77 \mathrm{~cm}^{-1}(\sim 130 \mu \mathrm{m} ; \sim 2.3 \mathrm{THz})$ are sensitive to the molecular shape and size and can be used as an indicator for the degree of molecular compactness. PAHs with elongated shapes are more flexible than compact symmetric PAHs of similar size, shifting their lowest out-of-plane band position to longer wavelengths compared to their compact symmetric cousins.

\subsection{PAH Charge}

Figure 8 shows the far-IR spectra of three large, compact PAHs $\left(\mathrm{C}_{66} \mathrm{H}_{20}, \mathrm{C}_{78} \mathrm{H}_{22}, \mathrm{C}_{96} \mathrm{H}_{24}\right)$ in their $-1,0,+1,+2$, and +3 charge states. The figure reveals that varying charge does not have a strong influence on band positions for this subset of large compact PAHs. Relative band intensities, however, do show variations. This effect is most pronounced for the $\mathrm{C}_{66} \mathrm{H}_{20}$ and $\mathrm{C}_{96} \mathrm{H}_{24}$ anions and $\mathrm{C}_{78} \mathrm{H}_{22}$ triply charged form. Interestingly, the absolute intensities for the triply charged form of $\mathrm{C}_{78} \mathrm{H}_{22}$ and the anion of $\mathrm{C}_{96} \mathrm{H}_{24}$ are nearly 10 times stronger than those for the other charged forms. However, comparing with density functional theory calculations using the Becke-Perdew86 (BP86; Becke 1988; Perdew 1986) functional suggests that the B3LYP results could be somewhat unreliable here, and therefore the differences smaller (see also Bauschlicher et al. 2010).

\subsection{Nitrogen Containing Compact PAHs (PANHs)}

The far-IR spectra of three large, positively charged PAHs, with varying degrees of nitrogen substitution and placement, are presented in Figure 9. The left panel in Figure 9 compares the far-IR spectrum of $\mathrm{C}_{54} \mathrm{H}_{18}^{+}$with the spectra of six different isomers in which one carbon atom is replaced with a nitrogen atom. The right panel in Figure 9 compares the far-IR spectrum of $\mathrm{C}_{96} \mathrm{H}_{24}^{+}$with the spectra of eight single nitrogen containing isomers. Remarkably, these figures show that, in general, band positions and absolute intensities are hardly affected by nitrogen substitution. The middle panel in Figure 9 compares the far-IR spectra of $\mathrm{C}_{54} \mathrm{H}_{18}^{+2}$ with the spectrum of eight different isomers in which two carbon atoms are replaced with two nitrogen atoms. Apart from introducing more activity in the $20-33 \mu \mathrm{m}$
Table 1

The Computed Frequencies and Integrated Cross-sections for the Lowest "Drumhead" Modes for the Coronene and Pyrene "Families"

\begin{tabular}{lcccccc}
\hline \hline \multicolumn{3}{c}{ Coronene "Family" } & & \multicolumn{3}{c}{ Pyrene "Family" } \\
\cline { 1 - 2 } \cline { 5 - 7 } Species & $\begin{array}{c}v \\
\left(\mathrm{~cm}^{-1}\right)\end{array}$ & $\begin{array}{c}\sigma_{v, \text { int }} \\
\left(\mathrm{km} \mathrm{mol}^{-1}\right)\end{array}$ & & Species & $\begin{array}{c}v \\
\left(\mathrm{~cm}^{-1}\right)\end{array}$ & $\begin{array}{c}\sigma_{v, \text { int }} \\
\left(\mathrm{km} \mathrm{mol}^{-1}\right)\end{array}$ \\
\hline $\mathrm{C}_{24} \mathrm{H}_{12}$ & 124.0 & 4.93 & & $\mathrm{C}_{16} \mathrm{H}_{10}$ & 210.0 & 7.30 \\
$\mathrm{C}_{54} \mathrm{H}_{18}$ & 54.6 & 2.33 & & $\mathrm{C}_{30} \mathrm{H}_{14}$ & 114.7 & 4.21 \\
$\mathrm{C}_{96} \mathrm{H}_{24}$ & 31.3 & 1.34 & & $\mathrm{C}_{48} \mathrm{H}_{18}$ & 72.8 & 2.62 \\
$\mathrm{C}_{150} \mathrm{H}_{30}$ & 16.8 & 0.92 & & $\mathrm{C}_{96} \mathrm{H}_{26}$ & 37.7 & 1.32 \\
\hline
\end{tabular}

(15-9 THz; $\left.500-300 \mathrm{~cm}^{-1}\right)$ region, the incorporation of two nitrogen atoms versus one into $\mathrm{C}_{54} \mathrm{H}_{18}^{+}$does not significantly affect band positions or intensities, with the exception of the spectrum in the second-to-last panel from the top, where the cross-section is about three to five times larger. In all cases prominent bands fall between 33 and $50 \mu \mathrm{m}\left(9-6 \mathrm{THz} ; 300-200 \mathrm{~cm}^{-1}\right)$ and near $160 \mu \mathrm{m}\left(1.8 \mathrm{THz} ; 60 \mathrm{~cm}^{-1}\right)$. A description of the effect that nitrogen incorporation has on PAH spectra in the mid-IR can be found in Mattioda et al. (2003) and Hudgins et al. (2005).

\section{A CLASSICAL DESCRIPTION OF THE PAH MOLECULAR VIBRATIONS IN THE FAR-IR}

\subsection{The Out-of-plane Bending Modes}

Figure 10 focuses on the lowest vibrational mode of four members of the disk-shaped coronene and rhombus-shaped pyrene "families." The frequencies and integrated cross-sections are given in Table 1. The plot in the lower left frame of Figure 10 demonstrates the shift to lower frequencies of the lowest "drumhead" mode as molecular size increases.

The vibrational frequencies calculated for the coronene and pyrene "families" are well fitted by the single expression:

$$
v=600\left(\frac{10^{-15}\left(\mathrm{~cm}^{2}\right)}{A}\right) \quad\left(\mathrm{cm}^{-1}\right) \text {. }
$$



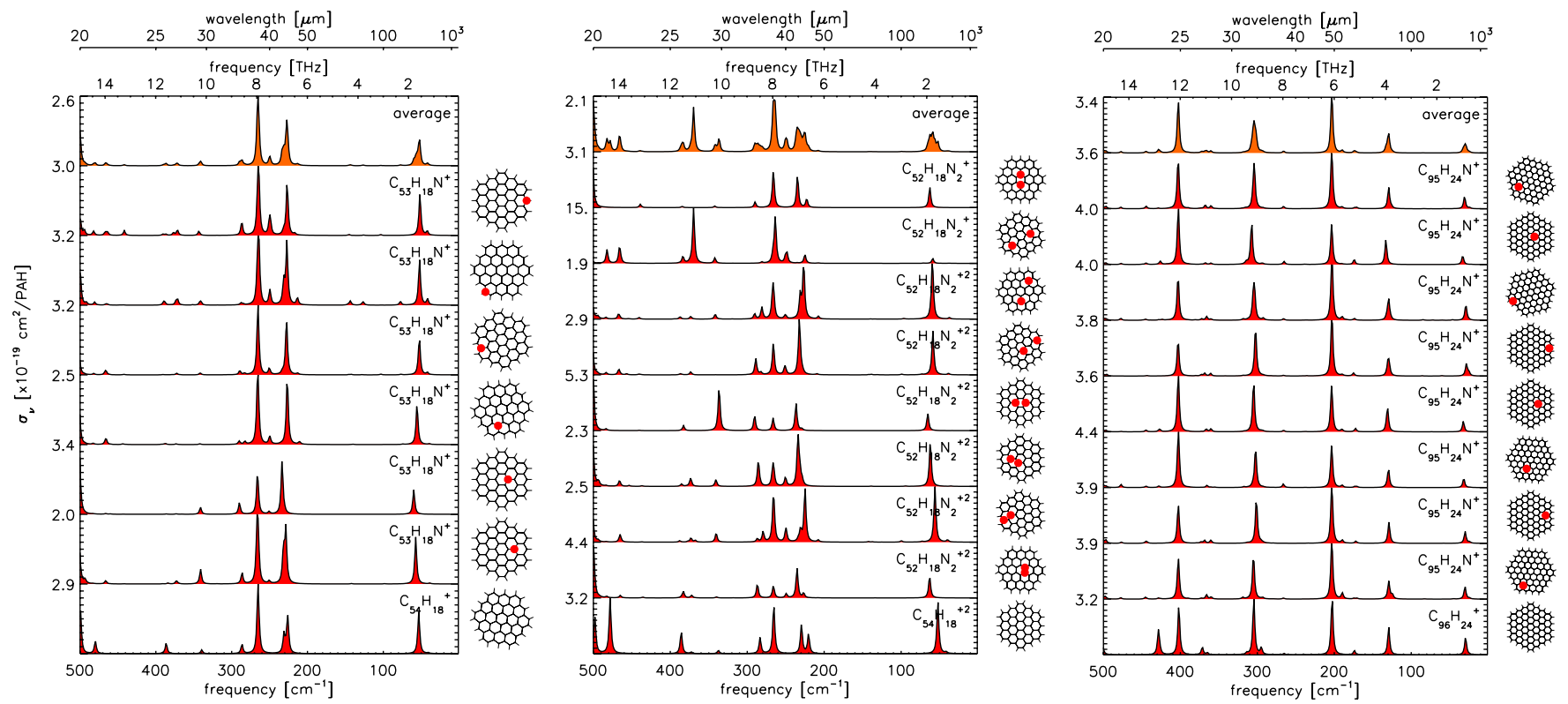

Figure 9. Computed far-IR $\left(500-1 \mathrm{~cm}^{-1} ; 15-0.03 \mathrm{THz} ; 20-10,000 \mu \mathrm{m}\right)$ absorption spectra of large compact, nitrogen containing PAHs and their equally weighted averages. Left: the spectrum of $\mathrm{C}_{54} \mathrm{H}_{18}^{+}$compared to the spectra of six different isomers in which one carbon atom is replaced with a nitrogen atom. Middle: the spectrum of $\mathrm{C}_{54} \mathrm{H}_{18}^{+2}$ compared to the spectra of eight different isomers in which two carbon atoms are replaced with two nitrogen atoms. Right: the spectrum of $\mathrm{C}_{96} \mathrm{H}_{24}^{+}$ compared to the spectra of eight different single nitrogen containing isomers. The positions of the substituted nitrogen are shown in red in the structures to the right of the spectra. Bands have been given Lorentzian profiles with an arbitrary FWHM of $3 \mathrm{~cm}^{-1}$.

(A color version of this figure is available in the online journal.)

The good agreement shows a systematic inverse dependence for the frequency of the lowest far-IR "drumhead" modes on PAH area. Clearly, the molecular geometric differences between the pyrene and coronene "families" do not play much of a role in determining the lowest "drumhead" mode frequency.

Considering the PAH molecule as a classical solid plate, it is perhaps not that surprising that area is far more important than geometry. The classic solution for the lowest "free-free" vibration of a solid plate follows from the general solution of the differential equation of motion after application of appropriate boundary conditions (Meirovitch 1997). The frequencies of "free-free" vibrating solid plates are then given by

$$
v_{m, n}=\frac{\pi}{2 c} \frac{1}{A} \sqrt{\frac{D}{\rho h}} \cdot\left(m^{2}+n^{2}\right),
$$

with $c$ being the speed of light, $A$ the area of the plate, $\rho$ the density of the plate, $h$ the plate thickness, and $D$ the flexural rigidity given by Equation (5). The modes are characterized by $m$ and $n$, the number of nodes along both plate axes. While this expression holds for square plates, geometry only enters weakly and the difference between squares and disks, where $m$ and $n$ count the number of sectorial and radial nodes, respectively, is of the order of $5 \%$ (Fan \& Luah 1993).

Because of a fundamental and commercial interest, there is a rich literature on the mechanical properties of graphene and carbon nano-tubes. Experimental studies using an atomic force microscope have demonstrated that the flexural rigidity as a function of thickness is well described by

$$
D=\frac{E h^{3}}{12\left(1-p^{2}\right)},
$$

with $E$ being Young's modulus and $p$ the Poisson ratio (Poot \& van der Zant 2008). However, calculating the flexural rigid- ity from a continuum model requires care and consistent choices of Young's modulus and effective thickness of the sheet (Shenderova et al. 2002). Good agreement between the continuum and discreet, atomistic, approach can be achieved when the two-dimensional nature of graphene is taken fully into account (Arroyo \& Belytschko 2004; Huang et al. 2006). The bending rigidity of a graphene sheet has been calculated to be $0.8-1.5 \mathrm{eV}$, depending on the method used (Salvetat et al. 2006). Adopting $7.5 \times 10^{-8} \mathrm{~g} \mathrm{~cm}^{-2}$ for the surface density $(\rho h)$, the data on the coronene and pyrene "families" are indeed well reproduced by the frequencies of like-sized graphene sheets (Figure 10). As expected, the agreement improves as the area increases and PAH size approaches that of graphene.

Table 2 predicts the radial, $n$, overtones of the coronene "family" extrapolated from the lowest "drumhead" mode, using Equation (4), and compares those to the frequencies found in the database. Figure 11 graphically compares the overtones with the predicted frequencies.

The data in Table 2 and Figure 11 demonstrate that, overall, PAH "drumhead" modes follow the classical approach well. Coronene, with the smallest area, shows the largest deviation from the $n=1$ and $n=2$ lines. Considering perhaps the contribution of the hydrogen atoms, where relative contribution to the total area diminishes as the molecule gets larger, might improve the comparison. Detailed inspection of the motions involved for these particular bands, shows that non-radial deformation occurs on the corners when the molecule gets sufficiently large. This may explain why the largest deviances between predicted and computed frequencies in the database are found for circumcircumcircumcoronene $\left(\mathrm{C}_{150} \mathrm{H}_{30}\right)$.

Figure 12 focuses on the lowest out-of-plane bending mode of four members of the acene "family," all bar-shaped molecules. The frequencies, integrated cross-sections and molecular lengths, are given in Table 3. Figure 12 shows the shift of the lowest "bar" mode to lower frequencies. In this case, 

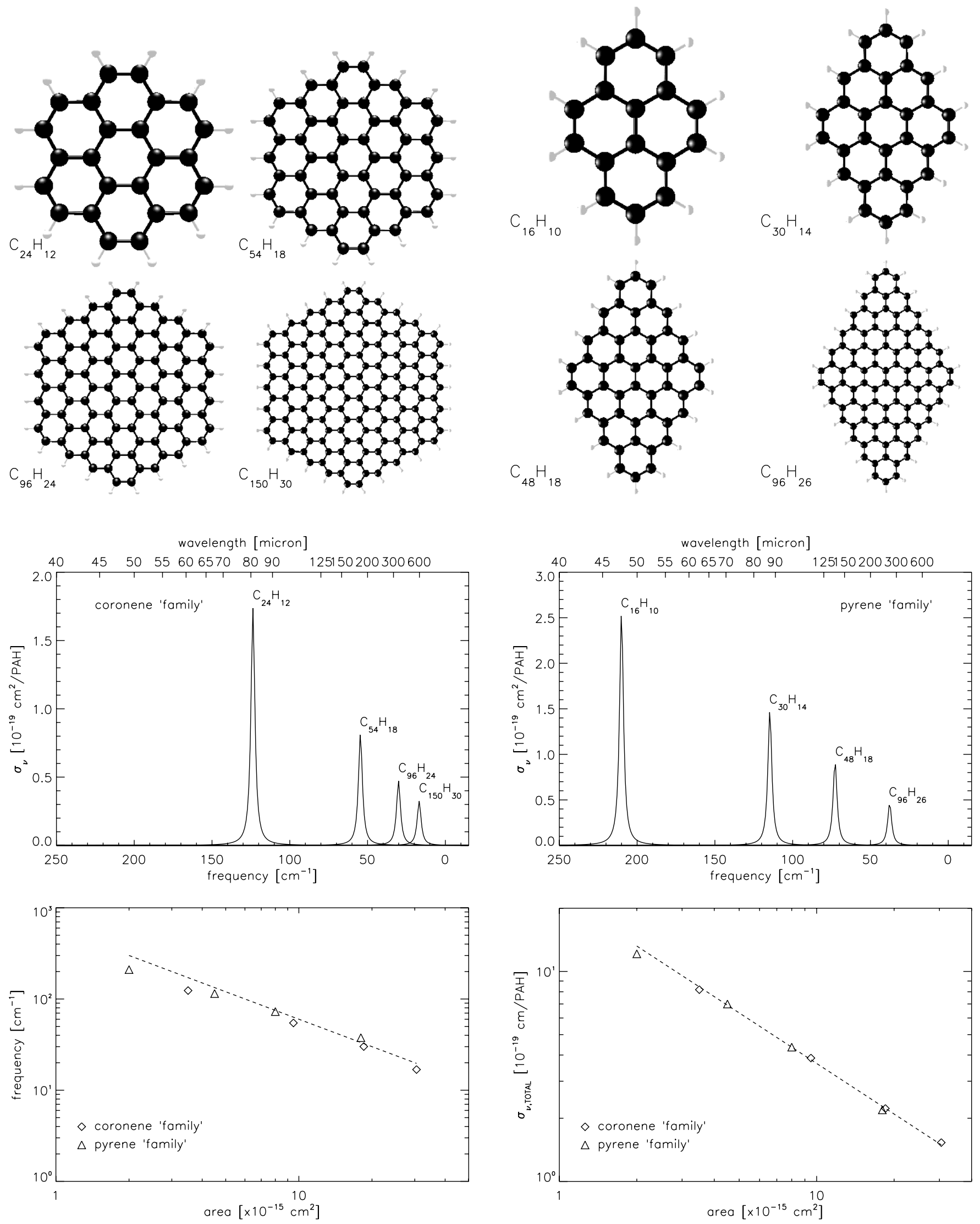

Figure 10. Shift of the "drumhead" modes to lower frequencies and lower intensity for four members of the coronene (top left) and pyrene (top right) "families." Middle: the shift of the lowest "drumhead" mode to lower frequencies as the molecule increases in size; left and right for the coronene and pyrene "families," respectively. Bands have been given Lorentzian profiles with an arbitrary FWHM of $3 \mathrm{~cm}^{-1}$. Bottom left: comparison of the predicted $(0,1)$ frequencies of a solid graphene plate (dashed line), i.e., Equations (3) and (4), with those for the coronene (diamonds) and pyrene (triangles) "families." Bottom right: integrated cross-section as a function of area for the coronene (squares) and pyrene (triangles) "families." The dashed line shows the least-squares fit represented by Equation (9). Note: circumcircumcircumcoronene $\left(\mathrm{C}_{150} \mathrm{H}_{30}\right)$ is not part of database version 1.11 , but will be included in a future release. 
Table 2

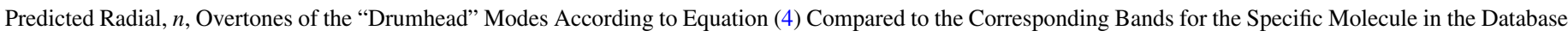

\begin{tabular}{|c|c|c|c|c|c|c|c|c|}
\hline \multirow[t]{2}{*}{$n$} & \multicolumn{2}{|c|}{ Coronene } & \multicolumn{2}{|c|}{ Circumcoronene } & \multicolumn{2}{|c|}{ Circumcircumcoronene } & \multicolumn{2}{|c|}{ Circumcircumcircumcoronene } \\
\hline & Predicted & Database & Predicted & Database & Predicted & Database & Predicted & Database \\
\hline 1 & 124.0 & & 54.6 & & 31.3 & & 16.8 & \\
\hline 2 & 496.0 & $548.6(11 \%)$ & 218.4 & $229.0(5 \%)$ & 125.2 & $129.8(4 \%)$ & 67.2 & $82.7(23 \%)$ \\
\hline 3 & $\ldots$ & $\ldots$ & 491.4 & $562.7(15 \%)$ & 281.7 & $303.4(8 \%)$ & 151.2 & $200.8(32 \%)$ \\
\hline 4 & $\ldots$ & $\ldots$ & $\ldots$ & $\ldots$ & 500.8 & $572.9(14 \%)$ & 268.8 & $353.4(31 \%)$ \\
\hline 5 & $\ldots$ & $\ldots$ & $\ldots$ & $\ldots$ & $\ldots$ & $\ldots$ & 420.0 & $578.4(38 \%)$ \\
\hline
\end{tabular}

Note. The deviation in percent is given in parentheses. Note. For $n=1$ the values have been reproduced from Table 1 .

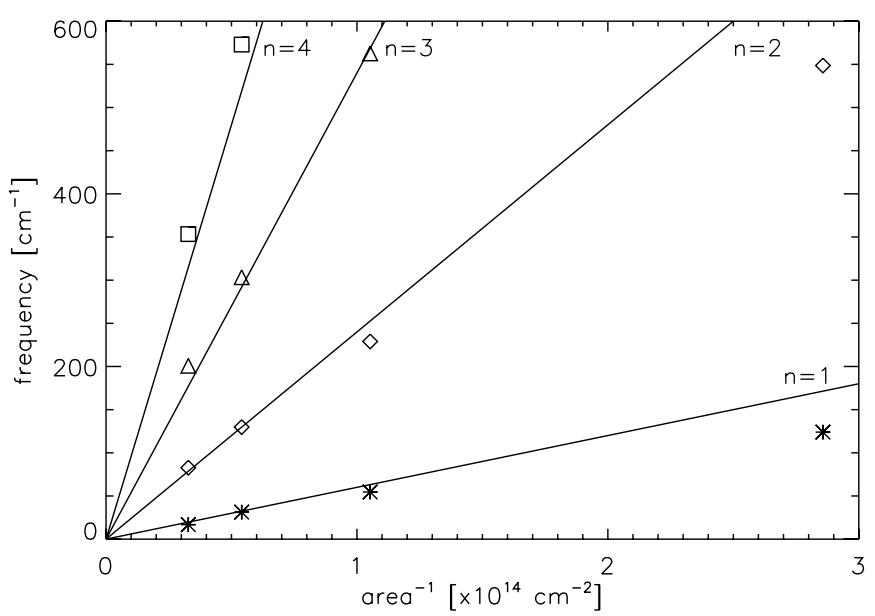

Figure 11. Overtones found in the database compared to those predicted by Equation (4) for $n=\{1,2,3,4\}$ (solid lines).

Table 3

The Computed Frequency and Integrated Cross-sections for the "Bar" Modes of the Acene "Family"

\begin{tabular}{lccc}
\hline \hline & Acene "Family" & \\
\hline Species & $\begin{array}{c}v \\
\left(\mathrm{~cm}^{-1}\right)\end{array}$ & $\begin{array}{c}\sigma_{v, \text { int }} \\
\left(\mathrm{km} \mathrm{mol}^{-1}\right)\end{array}$ & $\begin{array}{c}L \\
\left(10^{-8} \mathrm{~cm}\right)\end{array}$ \\
\hline $\mathrm{C}_{10} \mathrm{H}_{8}$ & 171.8 & 1.94 & 6.769 \\
$\mathrm{C}_{14} \mathrm{H}_{10}$ & 90.6 & 1.03 & 9.228 \\
$\mathrm{C}_{18} \mathrm{H}_{12}$ & 56.1 & 0.56 & 11.69 \\
$\mathrm{C}_{22} \mathrm{H}_{14}$ & 38.2 & 0.34 & 14.15 \\
\hline
\end{tabular}

Note. $L$ is the length, see Figure 12 .

with increasing molecular length $L(\mathrm{~cm})$, the frequencies are well fitted by the expression

$$
v=78\left(\frac{10^{-7}}{L}\right)^{2} \quad\left(\mathrm{~cm}^{-1}\right)
$$

where for the length $L, 2.46 \times 10^{-8} \mathrm{~cm}$ is taken as the length of a ring and $1.85 \times 10^{-8} \mathrm{~cm}$ is added for the protruding hydrogens on both ends, see the left frame in Figure 12.

The classic solution for the lowest "free-free" vibration of a bar follows also from the general solution of the differential equation of motion after application of appropriate boundary conditions (Den Hartog 1947; Pyykkö \& Zaleski-Ejglerd 2008). The lowest "free-free" vibrational frequency is then given by

$$
v=\frac{\pi}{4 c \sqrt{\frac{1}{4}-\frac{2}{\pi^{2}}}} \frac{1}{L^{2}} \sqrt{\frac{D}{\rho h}},
$$

with $c$ being the speed of light, $\rho$ the density of the bar, $h$ the bar thickness, and $D$ the flexural rigidity. The flexural rigidity is related to the second moment of inertia and depends on the degrees of freedom the material is allowed. Where for plates all degrees of freedom are available, for bars there is only one. Therefore,

$$
D=\frac{E h^{3}}{3\left(1-p^{2}\right)},
$$

differing a factor 4 with Equation (5).

Adopting $D=0.375(=1.5 / 4) \mathrm{eV}$ for the rigidity and again $7.5 \times 10^{-8} \mathrm{~g} \mathrm{~cm}^{-2}$ for the surface density $(\rho h)$ demonstrates that the data on the acene "family" are well reproduced by Equation (7) (Figure 12). The study done by Pyykkö \& Zaleski-Ejglerd (2008) on the acenes shows that the overtones also scale well with $L^{-2}$.

The analysis of the coronene/pyrene "families" and the acene "family" reveals that the lowest (out-of-plane) skeletal modes involving the entire molecule, i.e., the "drumhead" and "bar" modes, can be understood in terms of elastic vibrations. However, this imposes a sensitivity to shape, related to the available degrees of freedom. One "family" resembles plates, the other bars. This sensitivity has also been seen in the study by Ricca et al. (2010).

\subsection{Band Strength}

For the coronene and pyrene "families," the bottom right panel in Figure 10 presents the integrated cross-section as a function of area. A clear trend is apparent and is well fitted by the expression

$$
\sigma_{v, \text { int }}=23\left(\frac{10^{-15}}{A}\right)^{4 / 5}\left(\times 10^{-19} \mathrm{~cm}^{2}\right) .
$$

The decrease in the integrated cross-section of the lowest "drumhead" mode with increasing PAH area for these compact PAHs is striking. Utilizing the Mulliken population analysis (estimated atomic charges) obtained from density functional theory shows that charge accumulates at the edge of the PAH molecule in alternating negatively and positively charged rings. Thus, considering a PAH molecule as a vibrating charged disk cannot be used to model the band intensities. It appears that a full quantum mechanical treatment is necessary to obtain the trend.

\section{ASTROPHYSICAL CONSIDERATIONS}

With Herschel and SOFIA making sensitive spectroscopic observations in the far-IR, it is of interest to assess the impact PAH emission should have on this region of the spectrum. The PAH far-IR bands hold information about the astronomical 

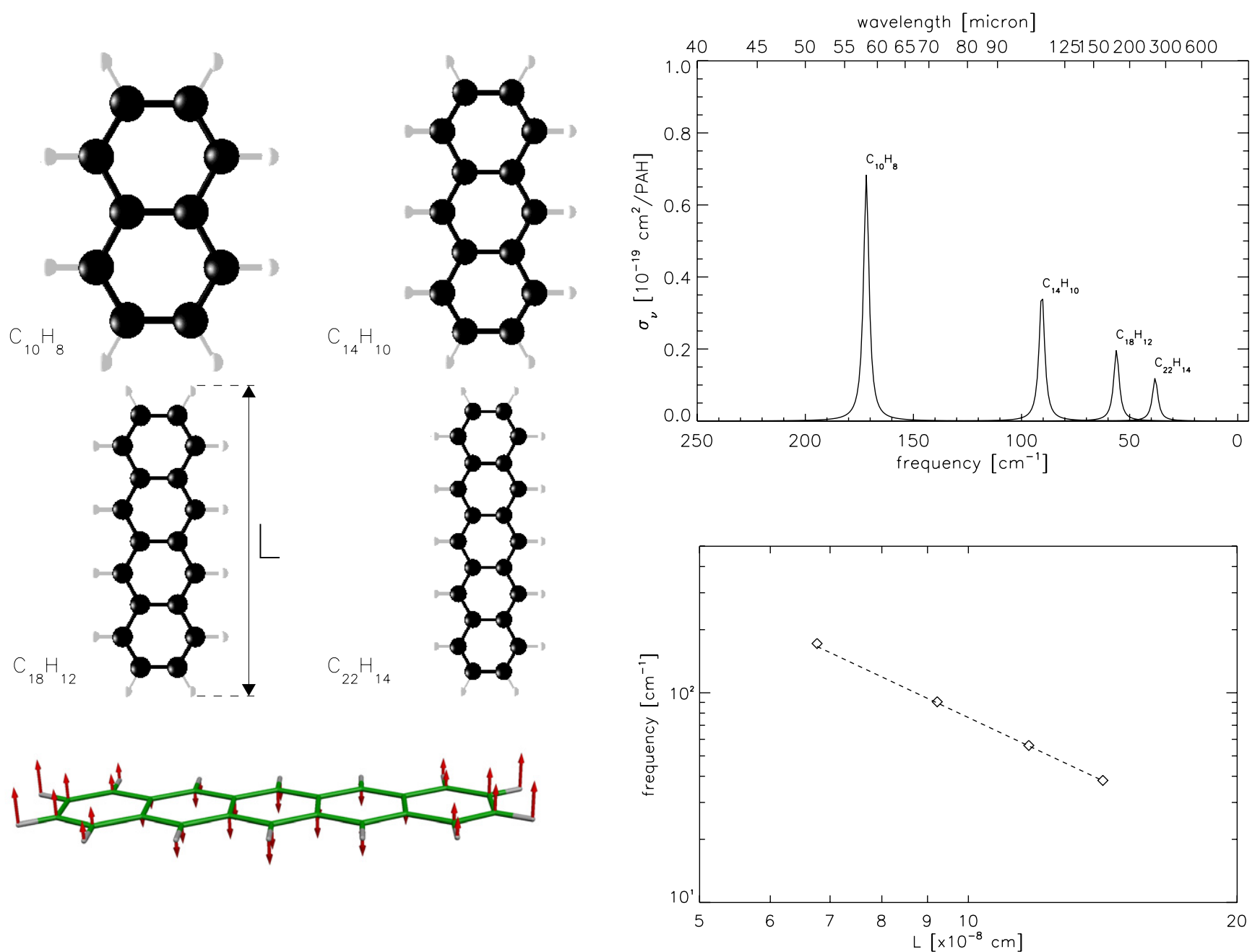

Figure 12. Top left: four members of the acene "family" and the definition of the lengths $L$ used in Equations (6) and (7), see Table 3. Bottom left: visualization of the lowest molecular vibration ("bar" mode) in pentacene $\left(\mathrm{C}_{22} \mathrm{H}_{14}\right)$. The carbon skeleton is shown in green, the hydrogen atoms in gray, and the displacement vectors in red. Top right: the shift of the lowest "bar" mode to lower frequencies as the acenes increase in size. Bands have been given Lorentzian profiles with an arbitrary FWHM of $3 \mathrm{~cm}^{-1}$. Bottom right: the frequency plotted vs. the length of the molecule compared to the predicted frequencies from Equation (7) (dashed line).

(A color version of this figure is available in the online journal.)

PAH family and the environment from which they emit that complements the information gleaned from the PAH mid-IR bands. Additionally, since PAH far-IR bands are weak and will likely blend with cold dust emission, it is important to consider an observing strategy. This section examines the far-IR emission process, evaluates the influence of $\mathrm{PAH}$ properties on their far-IR emission spectra, and provides some observational considerations based on Mulas et al. (2006a, 2006b).

\subsection{The PAH Far-IR Emission Process}

Far-IR absorption band strengths are generally an order of magnitude smaller than the mid-IR absorption band strengths. However, this can change significantly in the case of emission. Emission band intensities depend on vibrational energy content and this must be taken into account (see also the Appendix in Bauschlicher et al. 2010).

The PAHs that dominate the emission in the far-IR will attain a lower peak vibrational temperature than the relatively smaller PAHs that dominate the mid-IR upon absorption of the same visible/ultraviolet photon. In the thermal approximation this can be understood as an application of Wien's displacement Law, with the lower vibrational "temperature" shifting the peak of the emission into the far-IR.
The astronomical near/mid-IR PAH band ratios can be used to obtain a characteristic PAH excitation temperature. This vibrational temperature can then be used to approximate the emission spectrum from a given $\mathrm{PAH}$, which can be referred to as PAH emission at fixed temperature. While this approximation is a reasonable approach for analysis of the mid-IR bands, the fixed temperature approach fails when computing the total midto far-IR spectrum from a vibrationally excited PAH as its level of excitation cascades down. The effect of this cooling cascade on the total emission spectrum is particularly important for farIR bands because they are emitted near the end of the relaxation cascade.

When including the temperature cascade, conservation of energy leads to the following expression:

$$
4 \pi \sum_{i} \sigma_{i} \int_{T_{i}}^{T_{\max }} B\left(v_{i}, T\right)\left[\frac{d T}{d t}\right]^{-1} d T=h c v_{\mathrm{uv}}
$$

with the left-hand side describing the emission from the PAH and the right-hand side the exciting UV photon and where $\sigma_{i}$ is the absorption cross-section in vibrational mode $i, B\left(v_{i}, T\right)$ is Planck's function at frequency $v_{i}$ in mode $i$ at temperature $T, d T / d t$ is the cooling rate, and $v_{\mathrm{uv}}$ is the frequency of the 

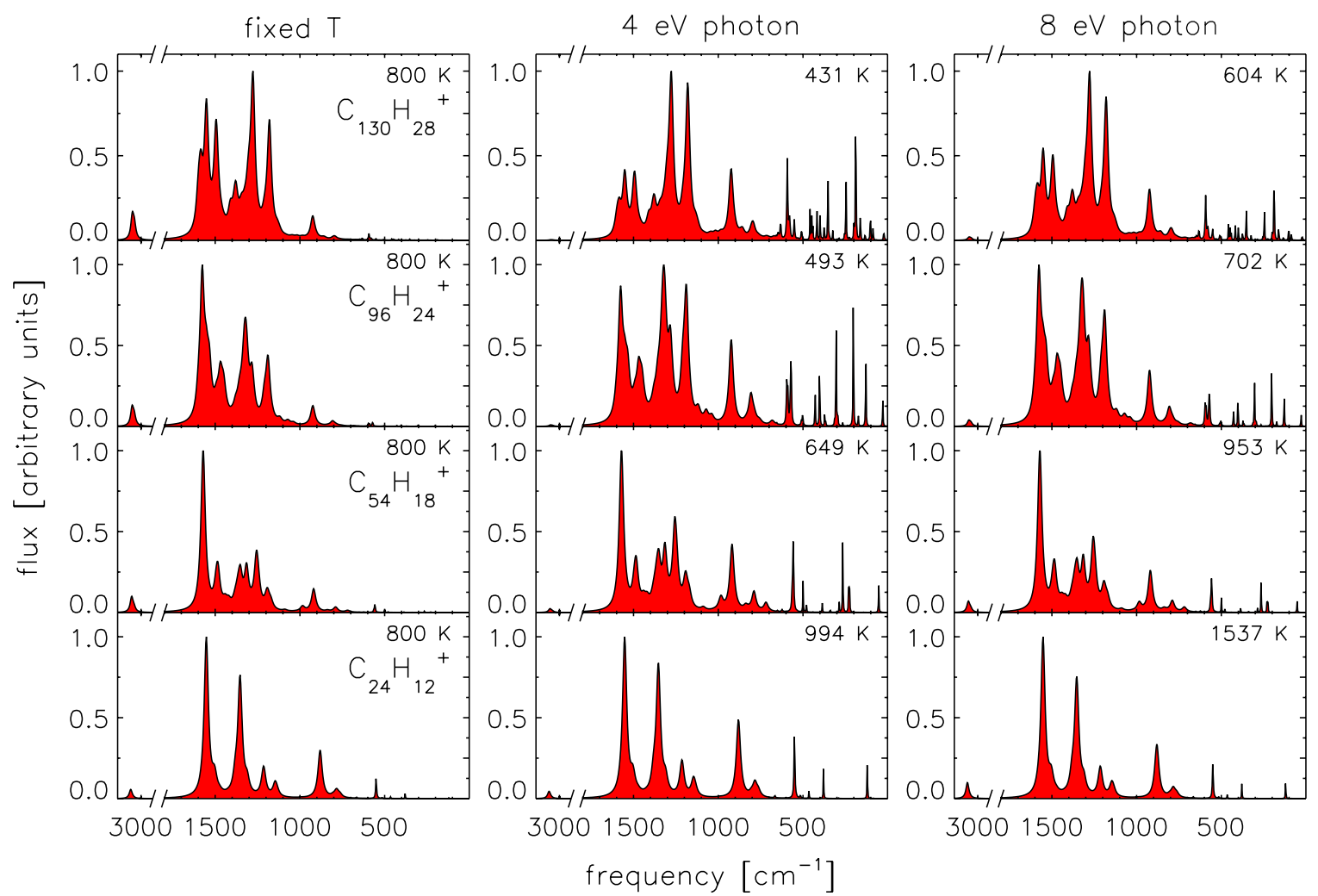

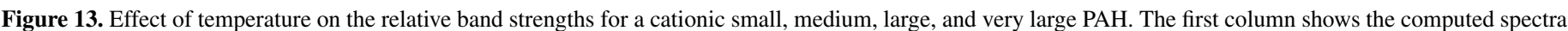

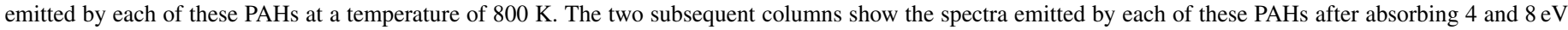

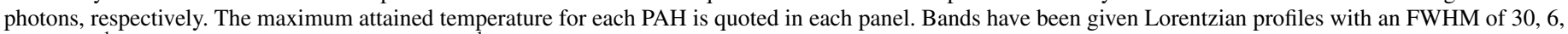
and $3 \mathrm{~cm}^{-1}$ for $3500-667,667-50$, and $50-1 \mathrm{~cm}^{-1}$, respectively (see the text for details).

(A color version of this figure is available in the online journal.)

absorbed UV photon. The sum is taken over all modes and the integral runs from the ambient PAH temperature $\left(T_{i}\right)$ up to the maximum attained temperature $\left(T_{\max }\right)$ of the $\mathrm{PAH}$ molecule upon absorption of the photon.

The maximum attained vibrational temperature is directly related to the energy of the absorbed photon through the PAH heat capacity $\left(C_{\mathrm{V}}\right)$, and in addition connects to the cooling rate:

$$
\frac{d T}{d t}=\left[\frac{d E}{d T}\right]_{\mathrm{V}}^{-1} \frac{d E}{d t}=\frac{4 \pi}{C_{\mathrm{V}}(T)} \sum_{i} \sigma_{i} B\left(v_{i}, T\right) .
$$

The heat capacity can be calculated by treating the PAH molecule as a molecular system of isolated harmonic oscillators or using analytical expressions estimated from experiment (see, e.g., Dwek et al. 1997). Bakes et al. (2001) provide an analytical approximation for the entire cooling rate.

Figure 13 demonstrates the difference between the emission spectrum from a specific $\mathrm{PAH}$ at a fixed temperature versus the total emission spectrum taking the full temperature cascade into account. The three spectra in each column show the emission from different sized PAHs computed at $800 \mathrm{~K}$ (left; fixed) and after absorbing a 4 and $8 \mathrm{eV}$ pumping photon (middle, right; cascade). Each row represents a different compact $\mathrm{PAH}$ size: from small, medium, large to very large $\left(\mathrm{C}_{24} \mathrm{H}_{12}, \mathrm{C}_{54} \mathrm{H}_{18}\right.$, $\mathrm{C}_{96} \mathrm{H}_{24}$ to $\mathrm{C}_{130} \mathrm{H}_{28}$ ).

Figure 13 illustrates two points. First, the excitation temperature that characterizes the mid-to-far-IR bands is much lower than that which characterizes the near-to-mid-IR bands. Thus, the ratio of the mid/far-IR bands can be much larger than expected on the basis of the average excitation temperature deduced from the near/mid-IR band ratios. Second, with increasing PAH size the far-IR emission becomes more important.

The FWHM of the profiles observed in astronomical mid-IR spectra varies substantially for the different wavelength regions. Emission features generally have an observed characteristic line width of $10-30 \mathrm{~cm}^{-1}$ for the 6-9 $\mu \mathrm{m}$ region (Peeters et al. 2004), while for the bands between 15 and $20 \mu \mathrm{m}$, a range between 4 and $8 \mathrm{~cm}^{-1}$ is more typical (e.g., van Kerckhoven et al. 2000; Moutou et al. 1998; Boersma et al. 2010). Due to the lack of observational data, emission band widths of astronomical PAHs in the far-IR are currently unknown. Furthermore, the shape and width of the emission profiles in the mid-IR are determined by anharmonicity, but in the far-IR it is the rotational $(P Q R)$ envelope that determines band profile and width. Estimating rotational profiles is an involved task because the rotational state of large PAHs is not well quantified (see, e.g., Rouan et al. 1992; Mulas 1998; Ysard \& Verstraete 2010). Usually several mechanisms are considered to transfer angular momentum to the PAH molecule, including the emission of IR photons. This then drives rotation, which can be expressed by a rotational temperature. It is this key parameter that determines the width of the profile. Large PAH molecules are likely to have a very low rotational temperature (angular momentum) due to their large inertial mass. However, some models suggest that the rotational temperature could vary significantly depending on the astrophysical region considered, ranging from tens to several hundreds of degrees Kelvin for a PAH 100 carbon atoms in size 
(e.g., Ysard \& Verstraete 2010). Others use general assumptions to arrive at a rotational band profile and band width (e.g., Mulas et al. 2006a). Such detailed considerations are beyond the scope of this paper, which focuses on general trends. Therefore, we use a Lorentzian band shape with an arbitrary FWHM of $3 \mathrm{~cm}^{-1}$. In such a favorable case, the narrow band width sharpens the spectral contrast with the mid-IR as can be seen from Figure 13.

\subsection{PAH Properties and Their Far-IR Spectra}

The aspects of the PAH far-IR spectroscopic properties presented in Sections 2 and 3 and possible impact on the observations are summarized below.

PAH shape. Figures 3 and 6 show that PAHs with single rings, whether as a pendent or a terminating ring, all have a band around $21 \mu \mathrm{m}$. This band shifts slightly with increasing size, which is best seen in the acenes.

Emission close to $21 \mu \mathrm{m}$ has been observed in several carbonrich post-asymptotic giant branch (AGB) stars and carbon-rich protoplanetary nebula that also show the mid-IR PAH features (Kwok et al. 1989; Geballe et al. 1992; Volk et al. 1999; Zhang et al. 2010). As pointed out by Volk et al. (1999), the presence of the mid-IR PAH emission features in these " $21 \mu \mathrm{m}$ sources" suggests that the carriers of the feature are related to carbon-rich aromatic compounds. The transitory nature of the astronomical $21 \mu \mathrm{m}$ band and the nature of these objects is consistent with the carriers being short-lived, carbonaceous species that are intermediates on the route to PAHs, PAH clusters, and amorphous carbon particles. In this regard, single rings can be considered PAH building blocks. Furthermore, the slight variation in peak position with size and charge evident in Figure 3 is similar to the observed peak position variations (Volk et al. 1999).

Since larger PAHs must grow from smaller PAHs, tracking the properties of the $21 \mu \mathrm{m}$ peak in carbon-rich stars as a function of age, along with the full spectrum of the mid-IR $\mathrm{PAH}$ features, may prove fruitful in gaining important insight into PAH formation and growth in carbon-rich AGB stars.

PAH size. As discussed in Section 3 for the PAHs in the pyrene and coronene "families," ranging in size from $\mathrm{C}_{16} \mathrm{H}_{10}$ to $\mathrm{C}_{150} \mathrm{H}_{30}$, molecular area (not shape) clearly plays the dominant role in determining the "drumhead" mode frequencies. Thus, although the spectral diversity between these different PAHs is small, the far-IR could provide a firm handle on the size (area) of the emitting PAHs and, thereby, put tight limits on the astronomical PAH size distribution. Excluding the more elongated structures (Ricca et al. 2010), this conclusion can be expanded beyond the pyrene and coronene "families," since it seems that these low-frequency modes can be approximated by a like-sized vibrating graphene sheet. Conversely, the small spectral diversity implies that identifying a unique $\mathrm{PAH}$ based on far-IR observations will require other means. Mulas et al. (2006a) point to the promise of resolving the ro-vibrational $P Q R$ band structure of the lowest PAH mode as such a means (see also Section 4.1). Ultimately, this would require knowledge of the rotational constants. While these have only been measured for a very few PAHs (Thorwirth et al. 2007), the constraints placed on the molecular identity by the profiles of the $P, Q$, and $R$ branches as well as their "congestion" could put tight constraints on the possible molecular geometries, making a unique identification plausible.

Dominant far-IR PAH bands. The well-known mid-IR emission features seem to be carried largely by compact PAHs, with structures similar to those considered in Figures 4 and 6 (Bauschlicher et al. 2008, 2009). Perusal of these two figures shows that species with about 100 carbon atoms in size have a band near $50 \mu \mathrm{m}\left(6 \mathrm{THz} ; 200 \mathrm{~cm}^{-1}\right)$. Indeed, the average spectra for these species, which is shown in the top frame of each set, have a strong, clear-cut emission band near $50 \mu \mathrm{m}$ $\left(6 \mathrm{THz} ; 200 \mathrm{~cm}^{-1}\right)$. While all of the average spectra in the top frame in Figures 8-6 show features clustering at several positions, most are not as prominent as the $50 \mu \mathrm{m}\left(6 \mathrm{THz} ; 200 \mathrm{~cm}^{-1}\right)$ band associated with compact PAHs of about 100 carbon atoms in size.

As demonstrated with the "Jumping-Jack" mode near $50 \mu \mathrm{m}$ $\left(6 \mathrm{THz} ; 200 \mathrm{~cm}^{-1}\right)$ in irregular-shaped PAHs in Figure 6 , the same vibrational modes in similar-shaped PAH molecules can produce emission at about the same frequency. Recently, Ricca et al. (2010) have also shown that other very large, compact PAHs ranging in size from $\mathrm{C}_{82} \mathrm{H}_{24}$ to $\mathrm{C}_{130} \mathrm{H}_{28}$ have a prominent band peaking between 45 and $50 \mu \mathrm{m}(7$ and $6 \mathrm{THz} ; 222$ and $200 \mathrm{~cm}^{-1}$ ).

Interestingly, the catalog of $2.3-196 \mu \mathrm{m} \quad(130-1.5 \mathrm{THz}$; $4347-51 \mathrm{~cm}^{-1}$ ) Infrared Space Observatory spectra of compact $\mathrm{H}$ II regions from Peeters et al. (2002) shows a band near $50 \mu \mathrm{m}$ $\left(6 \mathrm{THz} ; 200 \mathrm{~cm}^{-1}\right)$ for the sources that show the mid-IR PAH bands. But, sources that do not show the mid-IR PAH bands do not show a band near $50 \mu \mathrm{m}\left(6 \mathrm{THz} ; 200 \mathrm{~cm}^{-1}\right)$.

Assuming an astronomical PAH size of 100 carbon atoms dominating the far-IR, the presence of a strong PAH emission band near $50 \mu \mathrm{m}\left(6 \mathrm{THz} ; 200 \mathrm{~cm}^{-1}\right)$ in objects showing midIR PAH features is predicted. If a strong PAH far-IR band is detected at other frequencies it likely also arises from the "pileup" or overlap of bands from different, closely related carriers. This would then provide more insight into the size and make-up of the emitting PAHs.

Charge. Charge state does not seem to play an important role in determining PAH far-IR band positions. However, as shown in Figure 8, relative band intensities can change significantly as a function of a specific PAH charge state. Under favorable conditions where some bands of a specific PAH have been identified, the more extreme intensity changes with varying charge could be important in understanding emission from extended regions. In such regions, ionization states vary with position, giving rise to variations in relative band strengths as a function of distance from the exciting star, electron density, radiation field, etc.

Nitrogen containing PAHs (PANHs). The spectra presented in Figure 9 show that the inclusion of nitrogen in the PAH hexagonal structure of large compact PAHs has little effect on far-IR band positions. Except for the shift in the $\mathrm{C}-\mathrm{C}$ stretching band near $6.2 \mu \mathrm{m}$, similar behavior is found for the mid-IR bands (Mattioda et al. 2003; Hudgins et al. 2005). Thus, astronomical PAH far-IR emission features will likely provide no information regarding $\mathrm{PAH}$ nitrogen content.

Far-IR recipe. The analysis of the "Jumping-Jack" and "drumhead" modes of compact PAHs presented here shows that the frequencies and intensities vary with $\mathrm{PAH}$ area (size) in a predictable manner, i.e., Equations (1)-(3) and (9). Evenmore, the frequencies for the "drumhead" modes can be calculated using classical plate theory, i.e., Equation (4). However, the "drumhead" modes show surprising behavior for the intensity, which decreases with increasing molecular size. Although this trend is currently not understood, these expressions should prove useful to model far-IR PAH spectra. It should be noted that the PAHs 
in the coronene "family," due to their symmetry, do not possess a "butterfly" mode. Also, the non-pure-radial modes $(m>0$ in Equation (4)) carry no intensity due to a lack of a net dipole moment. Currently, studies are underway examining these trends for larger members of the coronene "family," up to 384 carbon atoms (A. Ricca et al. 2011, in preparation).

\subsection{Observational Considerations}

The detection of PAH far-IR features will likely be hampered by poor contrast with strong dust background emission as well as spectral confusion with other features and bands. On the other hand, the PAH far-IR emission features will likely have distinctive natural line widths from most other emitting species, relatively more energy will be radiated in the far-IR for large and cold PAHs compared to the warm PAHs dominating the mid-IR, and objects can be chosen to minimize the contribution of dust emission. Mulas et al. (2006a) have shown that the lowest vibrational transitions of interstellar PAHs with sizes $N_{\mathrm{C}} \sim 48$ should typically contain a few tenths of a percent of the absorbed far-UV energy. While this is only a tiny fraction of the total energy, one can enhance the expected spectroscopic contrast by searching for far-IR PAH features in warm photodissociation regions. Here, classical dust is warm, shifting the peak blackbody emission from the far-IR well into the mid-IR range.

While detection of these transitions might prove to be challenging, one could further focus on emission from particularly radiative intense environments, such as that of the planetary nebula, NGC 7027. Telescope sensitivity will not be an issue in these environments, and UV photolysis will have whittled the PAH population to their most stable forms, leaving only a very limited number of different species. In this case, band "pileup" would be expected. Given their high stability and likely contribution to the mid-IR (Bauschlicher et al. 2008; Mattioda et al. 2009), the compact structures making up the coronene and pyrene "families" are particularly interesting in this regard. However, care must be taken to avoid environments in which a PAH might absorb a second FUV photon before having de-excited from the first, as otherwise it will not have had time to emit in the far-IR and overall far-IR emission will be suppressed.

\section{SUMMARY AND CONCLUSION}

This work summarizes the far-IR spectroscopic properties of large PAHs containing about 100 carbon atoms. Using the theoretical spectra in the NASA Ames PAH IR Spectroscopic Database (http://www.astrochem.org/pahdb), the role that molecular shape, size, charge, and nitrogen inclusion have on the far-IR PAH spectra have been studied.

PAH far-IR spectral richness increases with increasing $\mathrm{PAH}$ size. The linear fused ring PAH series, the acenes, have very simple far-IR spectra, with a prominent band near $21 \mu \mathrm{m}$. Interestingly, this falls close to the transitory $21 \mu \mathrm{m}$ emission feature seen in carbon-rich, post-AGB stars suggesting acenes as PAH building blocks. The much larger irregular-shaped PAHs with pendent rings studied here show the band.

Introducing edge irregularities increases the number of far-IR bands. However, series of PAH families with the same overall compact structure, e.g., the circumcircumcoronene $\left(\mathrm{C}_{96} \mathrm{H}_{24}\right)$ "family," have common "Jumping-Jack" modes that overlap. This enhances band strengths and spectral contrast at certain frequencies, making these regions of particular interest to search for PAH far-IR emission features. In particular, PAHs of about
100 carbon atoms in size generally have bands between 45 and $55 \mu \mathrm{m}$. Since these are PAHs that likely also contribute to the mid-IR emission features, a band near $50 \mu \mathrm{m}$ is predicted.

Detailed analysis of the out-of-plane bending "drumhead" and "bar" modes has shown that the lowest frequency and overtones of this type of molecular vibration can be treated as a classical vibrating graphene sheet and bar, respectively. From this point of view, for a family of similar-sized compact PAHs, geometry has a negligible effect on the band positions. Thus, these modes cannot help distinguish between different molecules, but do reflect size. Overall, this non-dependence of shape on far-IR spectra will make a positive identification of specific PAHs challenging.

Charge state plays a negligible role in determining $\mathrm{PAH}$ farIR band positions, but can influence relative band intensities. Nitrogen inclusion, on the other hand, has little effect on far-IR band positions and only weakly influences band intensities as compared to the mid-IR.

The NASA Ames PAH IR Spectroscopic Database, which now includes the far-IR spectra of almost 600 PAHs, provides both a broad and a deep overview of PAH far-IR properties. However, important caveats must be kept in mind. Since the database is limited in diversity and size range, some classes of PAHs that are not represented in the database may be important astronomically. The absence of species containing more than 130 carbon atoms is particularly significant for the far-IR since these will be at the lowest excitation levels and therefore can only relax by emitting in the far-IR. Studies on these larger PAHs are currently underway (A. Ricca et al. 2011, in preparation).

An observing strategy was considered to optimize the chances of detecting unequivocal PAH far-IR emission. By observing highly excited regions showing the mid-IR PAH features, one probes environments where the PAH population is likely to be comprised of the most stable members of the astronomical PAH family, PAHs which are compact and symmetric. These will tend to have bands which pile up in narrow (far-IR) regions, particular near $50 \mu \mathrm{m}$ for PAHs with $\sim 100$ carbon atoms, increasing chances of detection. In addition, the difficulties associated by blending with broadband emission from classical dust particles will be minimized as the dust in these regions is hot and the bulk of their blackbody emission is shifted to the mid-IR.

This work was supported through NASA's Long Term Space Astrophysics, Astrobiology, Laboratory Astrophysics, The Spitzer Space Telescope Archival Research, NASA's Astronomy + Physics Research and Analysis (APRA; NNX07AH02G), and Herschel Laboratory Astrophysics Programs; the Netherlands Organization for Scientific Research (NWO) and by an appointment to the NASA Postdoctoral Program at the Ames Research Center, administered by Oak Ridge Associated Universities through a contract with NASA. Studies of interstellar PAHs at Leiden Observatory are supported through advanced- ERC grant 246976 from the European Research Council.

\section{REFERENCES}

Arroyo, M., \& Belytschko, T. 2004, Phys. Rev. B, 69, 115415

Bakes, E. L. O., Tielens, A. G. G. M., \& Bauschlicher, C. W. 2001, ApJ, 556, 501

Bauschlicher, C. W., Peeters, E., \& Allamandola, L. J. 2008, ApJ, 678, 316

Bauschlicher, C. W., Peeters, E., \& Allamandola, L. J. 2009, ApJ, 697, 311

Bauschlicher, C. W., et al. 2010, ApJS, 189, 341

Becke, A. D. 1988, Phys. Rev. A, 38, 3098 
Boersma, C., Bauschlicher, C. W., Allamandola, L. J., Ricca, A., Peeters, E., \& Tielens, A. G. G. M. 2010, A\&A, 511, A32

Den Hartog, J. P. 1947, Mechanical Vibrations (New York: McGraw-Hill)

Dwek, E., et al. 1997, ApJ, 475, 565

Fan, S. C., \& Luah, M. H. 1993, J. Sound Vib., 165, 85

Geballe, T. R., Tielens, A. G. G. M., Kwok, S., \& Hrivnak, B. J. 1992, ApJ, 387, L89

Huang, Y., Wu, J., \& Hwang, K. C. 2006, Phys. Rev. B, 74, 245413

Hudgins, D. M., Bauschlicher, C. W., \& Allamandola, L. J. 2005, ApJ, 632, 316

Kwok, S., Volk, K. M., \& Hrivnak, B. J. 1989, ApJ, 345, L51

Malloci, G., Joblin, C., \& Mulas, G. 2007, Chem. Phys., 332, 353

Mattioda, A. L., Hudgins, D. M., Bauschlicher, C. W., Rosi, M., \& Allamandola, L. J. 2003, J. Phys. Chem. A, 107, 1486

Mattioda, A. L., Ricca, A., Tucker, J., Bauschlicher, C. W., \& Allamandola, L. J. 2009, ApJ, 137, 4054

Meirovitch, L. 1997, Principles and Techniques of Vibrations (Englewood Cliffs, NJ: Prentice-Hall)

Moutou, C., Leger, A., \& D’Hendecourt, L. 1996, A\&A, 310, 297

Moutou, C., Sellgren, K., Leger, A., Verstraete, L., Rouan, D., Giard, M., \& Werner, M. 1998, in ASP Conf. Ser. 132, Star Formation with the Infrared Space Observatory, ed. J. Yun, L. Liseau (San Francisco, CA: ASP), 47

Mulas, G. 1998, A\&A, 338, 243
Mulas, G., Malloci, G., Joblin, C., \& Toublanc, D. 2006a, A\&A, 460, 93

Mulas, G., Malloci, G., Joblin, C., \& Toublanc, D. 2006b, A\&A, 456, 161

Peeters, E., Mattioda, A. L., Hudgins, D. M., \& Allamandola, L. J. 2004, ApJ, 617, L65

Peeters, E., et al. 2002, A\&A, 381, 571

Perdew, J. P. 1986, Phys. Rev. B, 33, 8822

Poot, M., \& van der Zant, H. S. J. 2008, Appl. Phys. Lett., 92, 063111

Pyykkö, P., \& Zaleski-Ejglerd, P. 2008, Phys. Chem. Chem. Phys., 10, 114

Ricca, A., Bauschlicher, C. W., Mattioda, A. L., Boersma, C., \& Allamandola, L. J. 2010, ApJ, 709, 42

Rouan, D., Leger, A., Omont, A., \& Giard, M. 1992, A\&A, 253, 498

Salvetat, J. P., Désarmot, G., Gauthier, C., \& Poulin, P. 2006, in Mechanical Properties of Individual Nanotubes and Composites (Lecture Notes in Physics, Vol. 677; Berlin: Springer), 439

Shenderova, O. A., Zhirnov, V. V., \& Brenner, D. W. 2002, Critical Reviews in Solid State and Materials Sciences, Vol. 27 (London: Taylor \& Francis), 227

Thorwirth, S., Theulé, P., Gottlieb, C. A., McCarthy, M. C., \& Thaddeus, P 2007, ApJ, 662, 1309

Tielens, A. G. G. M. 2008, ARA\&A, 45, 289

van Kerckhoven, C., et al. 2000, A\&A, 357, 1013

Volk, K., Kwok, S., \& Hrivnak, B. J. 1999, ApJ, 516, L99

Ysard, N., \& Verstraete, L. 2010, A\&A, 509, A12

Zhang, Y., Kwok, S., \& Hrivnak, B. J. 2010, ApJ, 725, 990 